\title{
Enumerating limit groups
}

\author{
Daniel Groves and Henry Wilton
}

\begin{abstract}
We prove that the set of limit groups is recursively enumerable, answering a question of Delzant. One ingredient of the proof is the observation that a finitely presented group with local retractions (à la Long and Reid) is coherent and, furthermore, there exists an algorithm that computes presentations for finitely generated subgroups. The other main ingredient is the ability to algorithmically calculate centralizers in relatively hyperbolic groups. Applications include the existence of recognition algorithms for limit groups and free groups.
\end{abstract}

Mathematics Subject Classification (2000). 20F10, 20F65.

Keywords. Limit groups, algorithmic properties.

A limit group is a finitely generated, fully residually free group. Recent research into limit groups has been motivated by their role in the theory of the set of homomorphisms from a finitely presented group to a free group, and in the logic of free groups. This research has culminated in the independent solutions to Tarski's problems on the elementary theory of free groups by Z. Sela (see [19], [20] et seq.) and O. Kharlampovich and A. Miasnikov (see [12], [13] et seq.). Sela's work extends to the elementary theory of hyperbolic groups [21].

We will be entirely concerned with finitely presentable groups. A class of groups $\mathcal{E}$ is recursively enumerable if there exists a Turing machine that outputs a list of presentations for every group $\mathcal{E}$. T. Delzant asked if the class of limit groups is recursively enumerable [22, I.13].

Theorem A (Corollary 3.7). The class of limit groups is recursively enumerable.

Addendum. In [4] and [9], [8] it is shown that the isomorphism problem is solvable for the class of limit groups. Therefore, one can improve the above to a list in which each (isomorphism class of) limit group appears once and only once (Delzant [22] calls this property recursive).

On the other hand, using Tietze transformations, one can easily adapt the list so that it contains each finite presentation of a limit group once and only once. 
To enumerate limit groups, our approach is to use the structure theory of limit groups developed in [13]. An equivalent structure theory is described in [19], which could also be used. Either way, two problems need to be solved. First, one needs to be able to compute presentations for finitely generated subgroups of limit groups. We call this property effective coherence. Secondly, one needs to be able to compute centralizers of elements in limit groups. To solve the second problem we use the relatively hyperbolic structure on limit groups found in [5] and [1]. Our solution to the first problem relies on local retractions.

D. Long and A. Reid [14] defined a group to have local retractions, or property LR, if every finitely generated subgroup is a retract of a finite-index subgroup. A finitely presented group with local retractions is coherent. Furthermore, one can compute presentations for subgroups.

Theorem B (Theorem 2.4). There exists an algorithm that, given a finite presentation for a group $G$ with local retractions and a finite set of elements $S$, outputs a presentation for the subgroup generated by $S$.

It is a remarkable fact that limit groups are finitely presented. It was proved in [24] that limit groups have local retractions. There is a lengthier proof that limit groups are effectively coherent using the theorem, also proved in [24], that iterated centralizer extensions are coset separable with respect to their vertex groups.

As an application of Theorem A, in Section 4 we prove the following theorem.

Theorem $\mathbf{C}$ (Theorem 4.1). There exists an algorithm that, given as input a presentation for a group $G$ and a solution to the word problem in $G$, determines whether or not $G$ is a limit group.

In Corollary 4.3, we deduce the existence of a similar recognition algorithm for free groups (pointed out to us by Gilbert Levitt).

This paper is the first of a series, in which we intend to prove algorithmic versions of Sela's results. Specifically, enumerating limit groups will be useful in the algorithmic construction of Makanin-Razborov diagrams over free groups.

Acknowledgements. The authors would like to thank Zlil Sela for many insightful and generous conversations, and also François Dahmani and Vincent Guirardel for pointing out Corollary 4.2 to us. Thanks also to Gilbert Levitt for drawing Corollary 4.3 to our attention, and to Martin Bridson for explaining the ideas of the paragraph before Theorem 3.5. Finally, thanks to the referee for their careful reading and useful comments. The first author was supported in part by NSF Grants DMS0504251 and DMS-0813863. 


\section{Effective coherence}

A finitely generated group is coherent if all of its finitely generated subgroups are finitely presented. We will be interested in the following algorithmic version of coherence.

Definition 1.1. A coherent group $G$ is effectively coherent if there exists an algorithm that, given a finite subset $S$ as input, outputs a presentation for the subgroup generated by $S$.

A class $\mathcal{E}$ of coherent groups is uniformly effectively coherent if there exists an algorithm that, given as input a presentation of a group $G \in \mathcal{E}$ and a finite set $S$ of elements of $G$, outputs a presentation for the subgroup of $G$ generated by $S$.

An appealing consequence of this property is that, under mild hypotheses, one can determine if a homomorphism to an effectively coherent group is injective.

Lemma 1.2. If a group $G$ is effectively coherent then there exists an algorithm that, given a presentation for a group $H$, a solution to the word problem in $H$, and $a$ homomorphism $f: H \rightarrow G$, determines whether $f$ is injective.

Proof. Given a presentation for the image of $f$ and a solution to the word problem in $H$, it is easy to check whether or not $f$ has a well-defined inverse and hence if $f$ is injective. Therefore, if $G$ is effectively coherent it is easy to check if $f$ is injective.

Remark 1.3. Even without a solution to the word problem in $H$, there is a Turing machine that will confirm in finite time if the homomorphism $f$ is injective. Indeed, if $f$ is injective then we know what the inverse to $f$ must be. By effective coherence, it is possible to compute a presentation for the image of $f$, and the inverse homomorphism exists if and only if the relations for $f(H)$ hold in $H$ (under the supposed inverse map). Even though the word problem for $H$ may be unsolvable, the words which are equal to 1 in $H$ are recursively enumerable. If there is an inverse homomorphism then the relations for $f(H)$ (interpreted as words in the generators for $H$ ) will eventually appear on this list.

Of course, if the word problem in $H$ is unsolvable then there will in general be no Turing machine which terminates if the map $f$ is not injective. For example, one cannot tell in general if the group $H$ is the trivial group.

It is immediate that a finitely generated subgroup of an effectively coherent group is effectively coherent. If $\mathscr{G}$ is a class of groups, denote by $S(\mathscr{G})$ the class of finitely generated subgroups of groups in $\mathscr{E}$. We are interested in effective coherence because it allows the property of being recursively enumerable to pass from $\mathcal{G}$ to $\delta(\mathscr{E})$. Furthermore, uniform effective coherence also passes to subgroups. 
Lemma 1.4. If $\mathcal{G}$ is recursively enumerable and uniformly effectively coherent then $S(\mathscr{G})$ is recursively enumerable and uniformly effectively coherent.

Proof. Enumerating the presentations of groups $G \in \mathcal{E}$ and finite subsets $S \subset G$, then using uniform effective coherence to compute presentations for $\langle S\rangle$, one enumerates presentations for every group in $S(\mathscr{E})$. So $\delta(\mathscr{E})$ is recursively enumerable.

Given a presentation for a group $G \in S(\mathscr{E})$ and a finite subset $S$ of $G$, enumerate groups $K \in \mathscr{E}$ and homomorphisms $f: G \rightarrow K .{ }^{1}$ Moreover, given a homomorphism $f: G \rightarrow K$, wait to see if the Turing machine described in Remark 1.3 shows that $f$ is injective. Since $G \in S(\mathscr{G})$, running the above tests in parallel one will eventually find such an injection $f$. Using the effective coherence of $K$, one can now compute a presentation for $\langle f(S)\rangle$. So $S(\mathcal{G})$ is uniformly effectively coherent.

We approach effective coherence through local retractions.

\section{Local retractions}

A group $G$ retracts onto a subgroup $H$ if the inclusion map $H \hookrightarrow G$ admits a leftinverse $\rho: G \rightarrow H$. The subgroup $H$ is called a retract and the map $\rho$ is a retraction. Following [14], a group has local retractions if every finitely generated subgroup is a retract of a finite-index subgroup. This has immediate consequences for coherence. Denote by $F_{X}$ the free group on a set $X$.

Lemma 2.1 ([23], Lemma 1.3). Let $G=\langle X \mid \mathcal{R}\rangle$ be a finitely presented group, and suppose that $\rho$ is a retraction from $G$ onto a subgroup $H$. Fix a lift of the retraction $\rho$ to a map $\rho^{\prime}: F_{X} \rightarrow F_{X}$. Then a (finite) presentation for $H$ is

$$
\left\langle X \mid \mathcal{R},\left\{x^{-1} \rho^{\prime}(x) \mid x \in X\right\}\right\rangle \text {. }
$$

Proof. Let $p: F_{X} \rightarrow G$ be the natural epimorphism and let $q=\rho \circ p: F_{X} \rightarrow H$. Denote the inclusion $H \hookrightarrow G$ by $i$ and note that $p \circ \rho^{\prime}=i \circ q$. The lemma follows directly from the claim that

$$
\operatorname{ker} q=\operatorname{ker} p\left\langle\left\langle\left\{x^{-1} \rho^{\prime}(x) \mid x \in X\right\}\right\rangle\right\rangle .
$$

Because $q=\rho \circ p$ it follows that ker $p \subset \operatorname{ker} q$. For any $x \in X$ we have

$$
q \circ \rho^{\prime}(x)=\rho \circ p \circ \rho^{\prime}(x)=\rho \circ i \circ q(x)=q(x)
$$

and hence $q\left(x^{-1} \rho^{\prime}(x)\right)=1$. So ker $p\left\langle\left\langle\left\{x^{-1} \rho^{\prime}(x) \mid x \in X\right\}\right\rangle \subset \subset \operatorname{ker} q\right.$.

For the reverse inclusion, note that if $g \in \operatorname{ker} q$ then $p \circ \rho^{\prime}(g)=1$ and hence $\rho^{\prime}(g) \in \operatorname{ker} p$. But it is clear that $g^{-1} \rho^{\prime}(g) \in\left\langle\left\langle\left\{x^{-1} \rho^{\prime}(x) \mid x \in X\right\}\right\rangle\right\rangle$ and hence $g=\rho^{\prime}(g)\left(g^{-1} \rho^{\prime}(g)\right)^{-1} \in \operatorname{ker} p\left\langle\left\langle\left\{x^{-1} \rho^{\prime}(x) \mid x \in X\right\}\right\rangle\right\rangle$ as required.

\footnotetext{
${ }^{1}$ One does not need a solution to the word problem for $K$ here: in parallel enumerate all maps of the generators of $G$ to $K$ and all possible proofs that a given map extends to a homomorphism. This will eventually find all homomorphisms.
} 
Corollary 2.2. Let $G$ be a finitely presented group. There is an algorithm that takes as input a finite presentation for $G$ and a collection of words which are the images of the generators under a homomorphism $\rho: G \rightarrow G$ that is a retraction onto $\rho(G)$, and outputs a presentation for $\rho(G)$.

We would like to thank the referee for pointing out that Lemma 2.1 was already proved by Wall in [23], and that a stronger version of Corollary 2.2 holds than we had in the previous version. We include a proof of Lemma 2.1 for completeness.

Since finite-index subgroups of finitely presented groups are finitely presented, coherence for finitely presented groups with local retractions follows immediately.

Proposition 2.3. If a finitely presented group $G$ has local retractions then $G$ is coherent.

By [14, Theorem 2.4], groups with local retractions are residually finite and hence have (uniformly) solvable word problem. Let $\mathscr{L} \mathcal{R}$ be the class of finitely presented groups with local retractions.

Theorem 2.4. The class $\mathscr{L} \mathcal{R}$ is uniformly effectively coherent.

Proof. Suppose we are given a finite presentation for a group $G \in \mathscr{L} \mathcal{R}$ and a finite collection of elements $S \subset \mathcal{E}$. Using the Reidemeister-Schreier Process (see, for instance, [15]), we can enumerate finite presentations for all finite-index subgroups $K$ of $G$. Since $G \in \mathscr{L} \mathcal{R}$, there is a finite-index subgroup $K$ of $G$ so that $\langle S\rangle \subseteq K$ and so that there exists a retraction $\rho: K \rightarrow\langle S\rangle$.

We find such a retraction as follows. In parallel, consider each of the finiteindex subgroups of $G$. Given such a finite-index subgroup $K$, look for the elements of $S$ as words in the generators for $K$. Suppose we have found a finiteindex subgroup $K$ so that $\langle S\rangle \subseteq K$, and a finite presentation $\langle X \mid R(X)\rangle$ of $K$, with $S=\left\{s_{1}(X), \ldots, s_{n}(X)\right\}$ written as words in $X^{ \pm}$. Now search for a collection of words $Y$ in $S^{ \pm}$with a bijection $\rho: X \rightarrow Y$ so that each of the relations of the form $R(Y)$ holds and so that for each $i$ we have $s_{i}(Y)=s_{i}(X)$. Then the map $\rho$ extends to a retraction $\rho: K \rightarrow\langle S\rangle$. Since there is a retraction, we will eventually find such a $K$ and $Y$.

The algorithm of Corollary 2.2 now computes a presentation for $\langle S\rangle$.

\section{Enumerating $\mathcal{I}$ and $\mathscr{L}$}

The class of iterated extensions of centralizers is defined inductively. If $G$ is a group, $g \in G$ and $Z(g)$ is the centralizer of $g$ then an amalgamated free product

$$
G^{\prime}=G * Z(g)\left(Z(g) \times \mathbb{Z}^{n}\right)
$$

is said to be obtained from $G$ by extension of centralizers. 
Definition 3.1. The class $\mathcal{I}$ of iterated extensions of centralizers is the smallest class of groups containing all finitely generated free groups which is closed under extension of centralizers. The class of limit groups is defined to be

$$
\mathscr{L}=S(\mathcal{I}),
$$

the class of finitely generated subgroups of iterated extensions of centralizers.

The usual definition of limit groups is as finitely generated fully residually free groups.

Definition 3.2. A group $G$ is fully residually free if, for every finite subset $X \subset G \backslash 1$, there exists a homomorphism $f: G \rightarrow F$, for a free group $F$, such that $1 \notin f(X)$.

A finitely generated group is fully residually free if and only if it is in $\mathscr{L}$, by a theorem of [13]. Fully residually free groups are residually finite (since free groups are) and so have solvable word problem. Using the fact that limit groups are fully residually free, the following fact is well known and easy to prove.

Lemma 3.3. If $G$ is a limit group and $g \in G$ then $Z(g)$ is a free abelian group.

By Theorem B of [24], limit groups have local retractions. It is clear that all groups in $\mathcal{I}$ are finitely presented.

Corollary 3.4. The class $\mathcal{I}$ is uniformly effectively coherent.

By Lemma 1.4, to enumerate limit groups it remains only to enumerate $\mathcal{I}$. The crucial step is the ability to calculate centralizers.

For this we use the relatively hyperbolic structure of limit groups (found independently by E. Alibegović [1] and F. Dahmani [5]). See [11] for an introduction to relatively hyperbolic groups (where in Farb's language we mean 'relatively hyperbolic with BCP'). Limit groups are torsion-free and hyperbolic relative to a finite collection of maximal noncyclic abelian subgroups. Dahmani [6] provides an algorithm which takes as input a finite presentation of such a relatively hyperbolic group and outputs a basis for a representative of each conjugacy class of noncyclic maximal abelian subgroup (Dahmani's algorithm takes as input an arbitrary finite presentation, and does not need to be given the 'relatively hyperbolic structure' of the group).

Another important tool will be the universal theory of a group. The elementary theory of a group $G$ is the set of all sentences in first-order predicate logic (possibly with coefficients) that hold in $G$. For example, $G$ is abelian if and only if the sentence

$$
\forall x, y \in G[x, y]=1
$$

is in the elementary theory of $G$. A universal sentence is a sentence in the elementary theory with a single universal quantifier. The universal theory of $G$ is the set of universal sentences in the elementary theory of $G$. 
In [16], Makanin proved that the universal theory of a free group $F$ is decidable that is, there exists an algorithm that, given as input a universal sentence, determines whether or not it lies in the universal theory of $F$. The universal theory of torsion-free relatively hyperbolic groups with abelian parabolic subgroups is also decidable, by another algorithm of Dahmani [7] (again the input is any finite presentation for the group, along with the universal sentence).

There is an alternative approach to calculating centralizers using biautomatic structures. It follows from work of Rebbechi [18] that limit groups are biautomatic, and the algorithm for finding automatic structures described in [10] can be adapted to find biautomatic structures [3]. In particular, one can calculate the fellow-traveller constant of the bicombing. Using the ideas of [2], it is then easy to compute a presentation for the centralizer of an arbitrary finite subset.

Theorem 3.5. There exists an algorithm that, given as input a presentation for a group $G \in \mathcal{I}$ and an element $g \in G$, outputs a minimal set of generators for $Z(g)$.

Proof. Apply Dahmani's algorithm from [6] to find a basis for a representative of each conjugacy class of maximal noncyclic abelian subgroup.

Let $g \in G$. There are two cases to consider: either $g$ is parabolic (which means conjugate into a noncyclic abelian subgroup) or else $g$ is hyperbolic (which means $g$ is not parabolic).

It is possible to decide whether or not $g$ is parabolic. This is because the universal theory of $G$ is decidable [7]. The element is parabolic if and only if there exists an element $h \in G$ so that $h g h^{-1}$ commutes with each element of one of the above bases for the noncyclic abelian subgroups. This is a existential sentence over $G$, which we can determine the truth of by Dahmani's algorithm from [7].

If $g$ is parabolic, then we will find such an element $h$, and the conjugates by $h^{-1}$ of the basis for the maximal noncyclic abelian subgroup generates the centralizer of $g$. In this case we have found a minimal generating set for $Z(g)$.

If $g$ is hyperbolic then its centralizer is generated by a maximal root of $g$. According to D. Osin [17], Theorem 1.16(3), it is possible to algorithmically extract roots from hyperbolic elements of $G$. On the face of it, Osin's algorithm needs to be given as input the relatively hyperbolic structure of the group. However, Dahmani's algorithm from [6] will find this structure, so we can make Osin's algorithm take only the finite presentation as input. Therefore, if $g$ is hyperbolic we can find a maximal root of $g$, and this maximal root is a minimal generating set for $Z(g)$.

Corollary 3.6. The set $\mathcal{I}$ is recursively enumerable.

Combining this with Corollary 3.4 it follows that the set of limit groups $\mathscr{L}$ is recursively enumerable, by Lemma 1.4.

Corollary 3.7. The set of limit groups $\mathscr{L}$ is recursively enumerable and uniformly effectively coherent. 


\section{Recognition algorithms}

Theorem 4.1. There exists an algorithm that, given as input a presentation for a group $G$ and a solution to the word problem in $G$, determines whether or not $G$ is a limit group.

Proof. Let $\mathcal{P}=\langle X \mid R\rangle$ be the finite presentation defining $G$. We have already noted that it is possible to enumerate all finite presentations of limit groups. Thus if $G$ is a limit group then $\mathcal{P}$ will eventually appear on this list.

Suppose then that $G$ is not a limit group. Then $G$ is not fully residually free, so there is a finite set $\left\{g_{1}, \ldots, g_{r}\right\}$ of nontrivial elements of $G$ so that for any homomorphism $\phi$ from $G$ to a free group $F$, at least one of the $g_{i}$ is in $\operatorname{ker}(\phi)$. This property of $G$ can easily be translated into a system of equations and inequations over $F$ as follows. Consider both the elements of $R$ and each $g_{i}$ as a word in $X^{ \pm}$, and write $R=\left\{r_{1}, \ldots, r_{k}\right\}$. Then the following sentence encodes the fact that at least one of $\left\{g_{1}, \ldots, g_{r}\right\}$ is in the kernel of any homomorphism from $G$ to $F$ :

$$
\forall X \subset F\left(r_{1}(X)=1 \wedge \cdots \wedge r_{k}(X)=1\right) \Rightarrow\left(g_{1}(X)=1 \vee \cdots \vee g_{r}(X)=1\right) .
$$

By Makanin's algorithm [16], it is possible to decide whether or not universal sentences are true in a free group. Enumerate finite sets of nontrivial elements of $G$ (the solution to the word problem allows us to know that the elements are nontrivial). Now, for each such finite set $\left\{g_{1}, \ldots, g_{r}\right\}$, decide whether the sentence (1) is true or not. If $G$ is not a limit group, we will eventually find a finite set for which (1) is true.

Of course, one cannot recognize limit groups amongst arbitrary finitely presented groups.

A cyclically pinched group is an amalgamated free product of two free groups with cyclic amalgamated subgroup. Some, but not all, of these groups are limit groups. In [22], I.3, Sela asks for necessary or sufficient conditions for a cyclically pinched group to be a limit group. We do not have an answer to this question. However, Theorem 4.1 implies that (at least in principle) one can decide whether or not a specific cyclically pinched group is a limit group. The following result was pointed out to us by François Dahmani and Vincent Guirardel (its proof contains the core of the proof of Theorem 4.1).

Corollary 4.2. There is an algorithm that takes as input a finite presentation of a cyclically pinched group and decides whether or not the defined group is a limit group.

It does not matter whether the input presentation exhibits the cyclically pinched nature of the group, since by applying some finite number of Tietze transformations it is possible to find such a presentation. Such a presentation gives an explicit solution to the word problem. Therefore Corollary 4.2 follows immediately from Theorem 4.1. 
As remarked above, limit groups are torsion-free and hyperbolic relative to their maximal abelian subgroups. There is an algorithm to distinguish free groups among such relatively hyperbolic groups; indeed, it is proved in [8], Theorem 1.4, that there exists an algorithm that computes the Grushko decomposition from a presentation of such a group. Combining this with Theorem 4.1, we obtain a similar recognition algorithm for free groups. This corollary was pointed out to us by Gilbert Levitt.

Corollary 4.3. There exists an algorithm that, given as input a presentation for a group $G$ and a solution to the word problem in $G$, determines whether or not $G$ is free.

One can also deduce a similar result for surfaces. In [9], Theorem D, it is shown that there exists an algorithm that computes a JSJ decomposition for a torsion-free, freely indecomposable group that is hyperbolic relative to its maximal abelian subgroups. In particular, combining this with the algorithm from [8], one can decide whether or not a limit group is a surface group. It follows as before that there exists an algorithm that, given as input a presentation for a group $G$ and a solution to the word problem in $G$, determines whether or not $G$ is a (fully) residually free surface group. (The only surface groups that are not residually free are the fundamental groups of the non-orientable surfaces of Euler characteristic 1, 0 and -1.)

\section{References}

[1] E. Alibegović, A combination theorem for relatively hyperbolic groups. Bull. London Math. Soc. 37 (2005), 459-466. Zbl 1074.57001 MR 2131400

[2] M. R. Bridson, On the subgroups of semihyperbolic groups. In Essays on geometry and related topics, Vol. 1, Monogr. Enseign. Math. 38, Enseignement Math., Geneva 2001, 85-111. Zbl 1053.20038 MR 1929323

[3] M. R. Bridson and L. D. Reeves, On the algorithmic construction of classifying spaces and the isomorphism problem for biautomatic groups. Preprint 2007.

[4] I. Bumagin, O. Kharlampovich, and A. Miasnikov, The isomorphism problem for finitely generated fully residually free groups. J. Pure Appl. Algebra 208 (2007), 961-977. Zbl 1121.20026 MR 2283438

[5] F. Dahmani, Combination of convergence groups. Geom. Topol. 7 (2003), 933-963. Zbl 1037.20042 MR 2026551

[6] F. Dahmani, Finding relative hyperbolic structures. Bull. Lond. Math. Soc. 40 (2008), 395-404. Zbl 05286359 MR 2418795

[7] F. Dahmani, Existential questions in (relatively) hyperbolic groups. Israel J. Math., to appear.

[8] F. Dahmani and D. Groves, Detecting free splittings in relatively hyperbolic groups. Trans. Amer. Math. Soc. 360 (2008), 6303-6318. Zbl 05374796 MR 2434288 
[9] F. Dahmani and D. Groves, The isomorphism problem for toral relatively hyperbolic groups. Publ. Math. Inst. Hautes Études Sci. (2008), 211-290. MR 2434694

[10] D. B. A. Epstein, J. W. Cannon, D. F. Holt, S. V. F. Levy, M. S. Paterson, and W. P. Thurston, Word processing in groups. Jones and Bartlett Publishers, Boston 1992. Zbl 0764.20017 MR 1161694

[11] B. Farb, Relatively hyperbolic groups. Geom. Funct. Anal. 8 (1998), 810-840. Zbl 0985.20027 MR 1650094

[12] O. Kharlampovich and A. Myasnikov, Irreducible affine varieties over a free group: I. Irreducibility of quadratic equations and Nullstellensatz. J. Algebra 200 (1998), 472-516. Zbl 0904.20016 MR 1610660

[13] O. Kharlampovich and A. Myasnikov, Irreducible affine varieties over a free group: II. Systems in triangular quasi-quadratic form and description of residually free groups. $J$. Algebra 200 (1998), 517-570. Zbl 0904.20017 MR 1610664

[14] D. D. Long and A. W. Reid, Subgroup separability and virtual retractions of groups. Topology 47 (2008), 137-159. Zbl 05544209 MR 2414358

[15] W. Magnus, A. Karrass, and D. Solitar, Combinatorial group theory. Interscience Publishers, New York 1966. Zbl 0138.25604 MR 0207802

[16] G. S. Makanin, Decidability of the universal and positive theories of a free group. Izv. Akad. Nauk SSSR Ser. Mat. 48 (1984), 735-749; English transl. Math. USSR-Izv. 25 (1985), 75-88. Zbl 0578.20001 MR 755956

[17] D. V. Osin, Relatively hyperbolic groups: intrinsic geometry, algebraic properties, and algorithmic problems. Mem. Amer. Math. Soc. 179 (843) (2006). Zbl 1093.20025 MR 2182268

[18] D. Y. Rebbechi, Algorithmic properties of relatively hyperbolic groups. PhD Thesis, Rutgers, 2001. arXiv:math/0302245

[19] Z. Sela, Diophantine geometry over groups I: Makanin-Razborov diagrams. Publ. Math. Inst. Hautes Études Sci. 93 (2001), 31-105. Zbl 1018.20034 MR 1863735

[20] Z. Sela, Diophantine geometry over groups II: Completions, closures and formal solutions. Israel J. Math. 134 (2003), 173-254. Zbl 1028.20028 MR 1972179

[21] Z. Sela, Diophantine geometry over groups VII: the elementary theory of a hyperbolic group. Proc London Math. Soc. Advance Access published February 23, 2009. doi:10.1112/plms/pdn052

[22] Z. Sela, Diophantine geometry over groups: a list of research problems. http://www.ma.huji.ac.il/ zlil/problems.dvi

[23] C. T. C. Wall, Finiteness conditions for CW-complexes. Ann. of Math. (2) 81 (1965), 56-69. Zbl 0152.21902 MR 0171284

[24] H. Wilton, Hall's theorem for limit groups. Geom. Funct. Anal. 18 (2008), 271-303. Zbl 1158.20020 MR 2399104

Received May 22, 2007; revised May 6, 2008 
D. Groves, MSCS UIC 322 SEO, M/C 249, 851 S. Morgan St., Chicago, IL 60607-7045, U.S.A.

E-mail: groves@math.uic.edu

H. Wilton, Department of Mathematics, 1 University Station C1200, Austin, TX 78712-0257, U.S.A.

E-mail: henry.wilton@math.utexas.edu 